\title{
Nutritional correlates of dietary glycaemic index: new aspects from a population perspective
}

\author{
Mandy Schulz ${ }^{1,2}$, Angela D. Liese ${ }^{2 *}$, Elizabeth J. Mayer-Davis ${ }^{2,3}$, Ralph B. D'Agostino $\mathrm{Jr}^{4}$, Fang Fang ${ }^{2}$, \\ Karen C. Sparks ${ }^{2}$ and Thomas M. Wolever ${ }^{5}$ \\ ${ }^{1}$ Department of Epidemiology, German Institute of Human Nutrition, Potsdam-Rehbruecke, 14558 Nuthetral, Germany \\ ${ }^{2}$ Department of Epidemiology and Biostatistics, Arnold School of Public Health, University of South Carolina, 800 Sumter Street, \\ Columbia, SC 29208, USA \\ ${ }^{3}$ Center for Research in Nutrition and Health Disparities, Arnold School of Public Health, University of South Carolina, Columbia, \\ SC 29208, USA \\ ${ }^{4}$ Department of Public Health Sciences, Wake Forest University School of Medicine, Winston-Salem, NC 27157, USA \\ ${ }^{5}$ Department of Nutritional Sciences, University of Toronto, Toronto, MSS 3E2, Canada
}

(Received 28 October 2004 - Revised 14 April 2005 - Accepted 21 April 2005)

\begin{abstract}
The role of dietary glycaemic index (GI) and glycaemic load (GL) in disease aetiology is of increasing interest. However, nutritional factors related to dietary GI and GL are not well understood from a population perspective. We aimed to investigate the relation ship between GI and GL and dietary intake at the food and nutrient level. Study subjects were 1071 non-diabetic adults from the Insulin Resistance Atherosclerosis Study, Exam I, 1992-4. Usual dietary intake was assessed with a 114-item modified Block food frequency questionnaire. Published GI values were assigned to food line items. Correlation and regression analyses were conducted. Intake of white bread, beer, meats and fries/fried potatoes was positively associated with average GI, as was fat, starch and alcohol intake (before and after energy adjustment). Intake of fruits and low-fat milk was inversely associated with GI, as were intakes of mono- and disaccharides, and fibre. GL was positively correlated with carbohydrate foods and inversely with non-carbohydrate foods. Gender-specific regression models identified eight food groups explaining $63 \%$ (men) and $55 \%$ (women) total GI variation after adjusting for demographics; $70 \%$ of variation in GL was explained by eleven (men) and nine (women) food groups, respectively. Although the GI of a food is an indicator of the ability of carbohydrates to raise blood glucose, dietary GI, unlike GL, appears to reflect more dimensions of diet than just carbohydrates, such as the combination of foods consumed. This may have implications for the interpretation of dietary GI in epidemiologic studies.
\end{abstract}

IRAS: Glycaemic index: Glycaemic load: Diet: Food groups: Nutrients

The concept of glycaemic index (GI) was introduced by Jenkins and Wolever in 1981 as a quantitative indicator of the ability of carbohydrates to raise blood glucose. The GI is defined as the incremental area under the glucose response curve following the intake of $50 \mathrm{~g}$ carbohydrate from a test food compared with the glucose area induced by the same amount of carbohydrate from a standard carbohydrate source, usually white bread or glucose (Jenkins et al. 1981). Originally, GI was primarily used in diabetes care to function as a physiological basis for food exchanges to account for differences in glycaemic potency of carbohydrates (Jenkins et al. 1983). Recently, the potential role of dietary GI in disease aetiology has become a topic of intense investigation in epidemiological studies (Augustin et al. 2002; Liese et al. 2004a).

Methodological issues including the determination of GI values for individual foods and mixed meals (O'Dea et al. 1980; Crapo et al. 1981; Vaaler et al. 1984; Englyst \& Cummings, 1986; Wolever \& Jenkins, 1986; Wolever et al. 1990; Ercan et al. 1994; Wolever \& Bolognesi, 1996) have been investigated exten- sively. However, the question of how the concept of the GI can be applied in epidemiological research and public health, where the entire diet needs to be considered, has received less attention. In the total diet, the relevance of a particular food's GI is qualified by the food's overall contribution to carbohydrate intake, i.e. its nutrient composition and frequency of consumption. To take the amount of carbohydrates consumed into account, the glycaemic load (GL) has been introduced, which is the amount of carbohydrate multiplied by its GI. The GL thus provides a measure of the interaction of GI and the amount of carbohydrates and thus expresses both the quality and the quantity of carbohydrates (Salmerón et al. 1997b). In the total diet of free-living individuals, this measure may be even more important than the GI, since the carbohydrate content of diet may vary substantially.

Understanding the potential impact of dietary GI and GL on health outcomes requires consideration of food and nutrient intake of the overall diet associated with the range of GI and GL values observed in free-living individuals. However, 
only limited research has described nutritional factors related to dietary GI and GL at the population level, and this research has primarily taken place in demographically homogeneous populations (Salmerón et al. 1997a; Buyken et al. 2001; Schulze et al. 2004). Therefore, the specific aims of the present study were to advance the understanding of nutritional correlates, both at the level of foods and nutrients, of dietary GI and GL in a multi-ethnic population of middle-aged adults among whom dietary intake has been assessed with a validated food frequency questionnaire (FFQ) that has been used extensively in nutritional epidemiological research (Mayer-Davis et al. 1997b, 1999, 2002; Sanchez-Lugo et al. 1997; Liese et al. 2003).

\section{Subjects and methods}

\section{Study population}

The design of the Insulin Resistance Atherosclerosis Study (IRAS) has been described in detail elsewhere (Wagenknecht et al. 1995). More than 1600 participants were recruited at four clinical centres between 1992 and 1994 for the IRAS baseline exam. The goal was to obtain nearly equal representation of participants across glucose tolerance status (normal; impaired glucose tolerance; non-insulin-taking type 2 diabetes mellitus); race/ethnicity (African American, Hispanic and non-Hispanic white); gender; age (40-49 years, 50-59 years, 60-69 years). Race/ethnicity was determined by self-report using 1990 US census questions. Two of the clinical centres (Los Angeles, CA and Oakland, CA) recruited African American (about two-thirds of subjects recruited) and non-Hispanic white participants (about one-third of subjects recruited). The other two clinical centres (San Luis Valley, CO and San Antonio, TX) recruited Hispanic (about two-thirds of recruited subjects) and non-Hispanic white participants (about one-third of recruited subjects). The final sample comprised 1624 people, of whom $38 \%$ were non-Hispanic white, $34 \%$ Hispanic and $29 \%$ African American; $44.2 \%$ (718) had normal glucose tolerance, $22.7 \%$ (369) had impaired glucose tolerance and $33.1 \%$ (537) had type 2 diabetes. All participants provided written informed consent as approved by their respective field centre's institutional review board.

\section{Dietary assessment}

Usual intake of foods and nutrients was assessed by interview using a 1-year, semi-quantitative 114-item FFQ interview modified from the National Cancer Institute Health History and Habits Questionnaire to include regional and ethnic food choices across the four clinical centres. Participants were asked to recall usual intake of foods and beverages over the past year. Both the frequency of intake and the serving size were ascertained. For analyses, the servings per day were standardized to the medium serving size by multiplying the intake frequency with the serving size after applying a weighing factor (small, 0.5; medium, 1.0; large, 1.5). For descriptive and analytical purposes we furthermore collapsed the 114 line items of the FFQ into thirty-three food groups based on similarities in food and nutrient composition, and computed the number of daily intake servings for each of these food groups. A description of the food groups has recently been published elsewhere (Liese et al. 2004b). The IRAS FFQ has been validated in a subset of the IRAS population (Mayer-Davis et al. 1999). Comparable validity and reproducibility of this instrument were demonstrated for the African
American, Hispanic and non-Hispanic white populations. Pearson correlation coefficients adjusted for total energy were 0.33 for sucrose, 0.37 for total carbohydrates and 0.46 for starch.

Alcohol intake was evaluated separately using a frequency approach with additional questions about recent use and average lifetime use. Subjects were asked about their usual consumption of wine (red and white wine), beer, mixed drinks/mixers and liquors. Frequency of consumption was expressed as servings per day standardized to a medium serving size. The nutrient database (HHHQ-DIETSYS analysis software, version 3.0; NCI, Bethesda, MD, USA), which was expanded to new foods and to include several additional nutrients, was used to estimate energy and nutrient intakes from the FFQ and the alcohol questionnaire.

We assigned mean GI values based on the white bread standard to all 114 FFQ line items plus three items assessed in the Exam 1 interview on alcohol consumption (beer, wine, liquors) plus several extra foods (foods that were reported to be consumed more than once per week in addition to line items queried in the FFQ) using information from the international tables (FosterPowell et al. 2002) and other available resources (T. M., personal communication). In the case of multiple line items (i.e. foods) per FFQ line, we assigned a GI value to each food and estimated the GI of the line using the weighted average of GI values based on prevalence of estimated population consumption of those items (Block et al. 1986; Mayer-Davis et al. 1999). Food items containing negligible amounts of carbohydrates (chiefly meat, fish, fats and some vegetables) were assigned a GI value of 100 , which will not greatly affect the estimated daily GI due to small amounts of carbohydrates consumed with these foods, but allows for the fact that whatever carbohydrate to be found in meats or fish is glycogen (Jenkins et al. 1981; Wolever et al. 1985, 1994). Cheese was assigned the GI of milk.

Average dietary GI was computed by summing the products of the digestible carbohydrate (i.e. total carbohydrate minus dietary fibre) content per serving for each item, multiplied by the average number of servings of that food per day, multiplied by its GI, all divided by the total amount of digestible carbohydrate daily intake (Wolever et al. 1994). The average dietary GL was computed as the GI but without dividing by the total digestible carbohydrate intake. Several additional issues inherent in the assignment of GI values to foods are worth mentioning. If for a given food more than one GI value was available, we initially considered all values. In a review process, one of us (T. W.) evaluated the methodology and specifics of the published GI-testing studies. We chose to consider as eligible only studies testing foods of Northern American formulation, if formulations from different countries were tested (as these may differ from country to country) and studies using standardized analytical methods. If more than one study was considered eligible for estimating the GI of a food, the simple mean of the GI was calculated. The GI of a mixed dish was determined by its major source of carbohydrates (e.g. spaghetti with tomato sauce was assigned the GI of plain spaghetti, sushi was assigned the GI of plain rice). The rationale for this proceeding was twofold: first, the recipe of some mixed dish line items was the mixed dish itself without provision of weights of the ingredients (e.g. spaghetti with tomato sauce); second, the infrequent consumption of other mixed dishes in our study population was assumed to be of minor importance for the estimation of dietary GI. For the purpose of presentation, dietary GI and GL were converted to the glucose $=100$ scale by multiplication with the factor 0.7 . 


\section{Statistical analysis}

We limited our analyses to 1071 individuals with normal (67\%) or impaired $(33 \%)$ glucose tolerance, excluding individuals with previously or recently diagnosed diabetes at baseline and incomplete dietary information.

Analyses were conducted for total energy, dietary fat (total fat, saturated fatty acids, MUFA and PUFA), dietary carbohydrates (total carbohydrates, fructose, sucrose, lactose, glucose, galactose, starch, fibre), protein and alcohol, and for the thirty-three food groups. Mean nutrient and food group intakes were calculated by quintiles of GI and GL. Since the distributions of most of the nutrients and food groups were positively skewed, log-transformed values were used in subsequent analyses. The natural logarithm of the nutrient alcohol and galactose and of all food groups was computed after adding 1 to each value to account for some individuals having zero intakes in these variables and the log of zero being undefined. To account for total energy intake, food group and nutrient intakes were adjusted using the residual method (Willett \& Stampfer, 1986).

In correlation analyses, the linear relationships between daily food group as well as nutrient intakes and dietary GI and GL were evaluated. Correlation coefficients were first estimated for the crude relationship between dietary factors and GI and GL. Next, adjusted correlations were estimated that took into account total energy intake. In these correlation analyses, we used the residuals of food group and nutrient intakes and dietary GI and GL. Subsequently, stepwise linear regression models were fit to detect the most predictive food groups to explain inter-individual variation in dietary GI and GL. There were forty-one independent variables (thirty-three food groups plus eight demographic covariates forced to be in the model including age, and seven indicator variables for ethnicity and clinic). The significance level for entry into the model was set at $P \leq 0 \cdot 1$. Food groups explaining less than $1 \%$ of variation in GI or GL were omitted due to negligible clinically relevant contribution to GI and GL variation. There were no marked differences in the selection of predictive food groups if the analysis was stratified by ethnicity, clinic or diabetes status (normal $v$. impaired glucose tolerance). However, we detected some differences by gender and thus regression models were run for men and women separately, controlled for age, ethnicity and clinic. We used Statistical Analysis Systems version 8.2 (SAS Institute Inc., Cary, NC, USA) for the statistical analysis.

\section{Results}

Descriptive characteristics of the study population are presented in Table 1. Focusing on demographic characteristics, subjects in the upper quintile of GI and GL were younger than subjects in lower quintiles. The proportion of women decreased across GI and GL quintiles. The relative proportion of Hispanics increased with increasing GI and GL quintiles, while the proportion of nonHispanic whites and African Americans decreased across quintiles in the Californian centres. This indicates that regional differences may exist and that Hispanics consume a diet that is on average of higher GI and GL compared with diets consumed by non-Hispanic whites and African Americans. Having less than high-school education was associated with high dietary GI and GL, as was current smoking status.

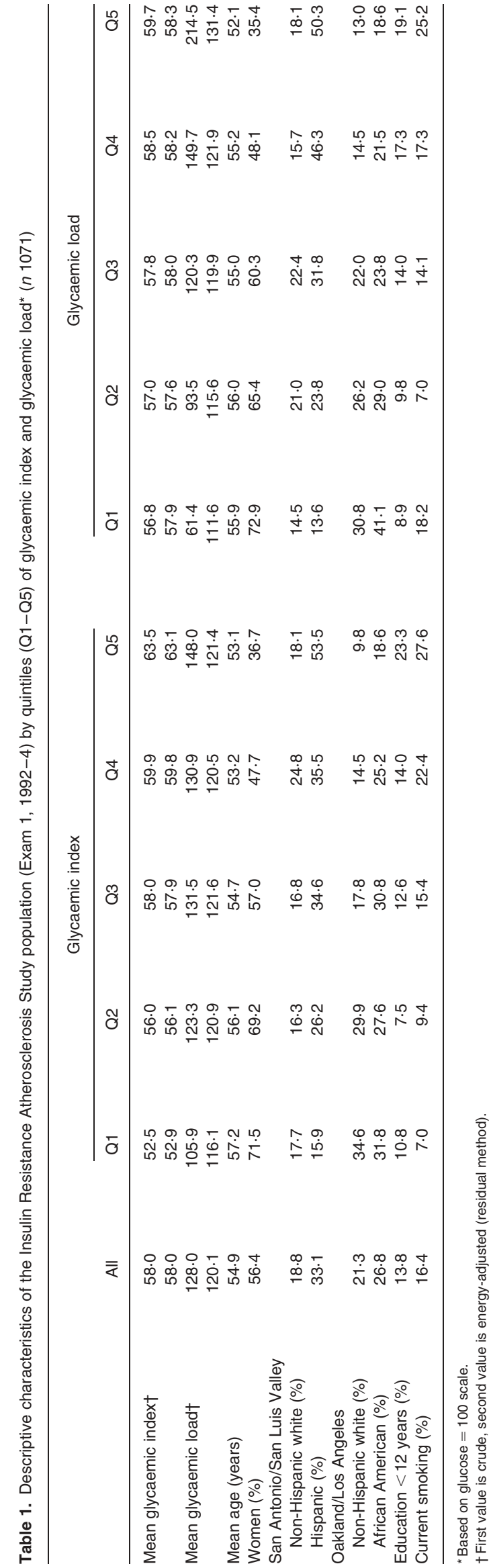




\section{Food groups}

We related all thirty-three food groups to dietary GI and GL, showing the average number of servings per day across GI and GL quintiles as well as correlation coefficients between number of servings of food groups and GI and GL, respectively (Table 2). Focusing on dietary GI, the food groups dark bread, rice/pasta, cruciferous vegetables and other vegetables, fruits, fruit juices, cottage cheese, low-fat milk, fish, poultry, diet soft drinks, ice cream, tofu and wine were gradually decreasing across GI quintiles and showed significant negative correlation with GI, even after adjustment for total energy. The food groups white bread, salty snacks, potatoes, fried potatoes, cheese, dried beans, eggs, meat, soft drinks, pastry, sweets, fats/ oils, coffee/tea, beer and mixed drinks were steadily increasing as dietary GI increased and were significantly positively correlated with dietary GI. The correlation was attenuated for potatoes, cheese, pastry and sweets after adjustment for total energy.

Focusing on dietary GL, consumption of all but five food groups was gradually increasing with increasing GL and also showed significant positive correlation with GL (Table 2); the strongest correlations were observed for white bread (0.65) and meat (0.56). Wine was the only food group with a negative correlation. Correlation coefficients were markedly attenuated for all food groups after controlling for total energy, leaving significant correlations for few food groups. For the food groups cheese, eggs, meat and fats/oils, the direction of the correlation was even reversed showing now significantly inverse correlations, i.e. that under isoenergetic conditions a high-GL diet is lower in cheese, eggs, meat and fats compared with a low-GL diet. This effect may not have emerged due to collinearity between total energy intake and the mentioned food groups (variance inflation factors $<10$ ). There were no marked gender differences in the correlations between food group intake and dietary GI or GL.

\section{Nutrients}

Focusing on dietary GI, total fat as well as fatty acids, digestible carbohydrate and starch intakes steadily increased from lowest to highest GI quintile, while sugars (mono- and disaccharides) and fibre intakes decreased with increasing GI (Table 3). Notably, after adjusting for total energy intake the direction of

Table 2. Mean food group intake by glycaemic index (GI)† and glycaemic load (GL)† quintiles (Q1-Q5)‡ and correlation coefficients between dietary Gl and GL and food groups $(\log ($ food group +1$))(n 1071)$

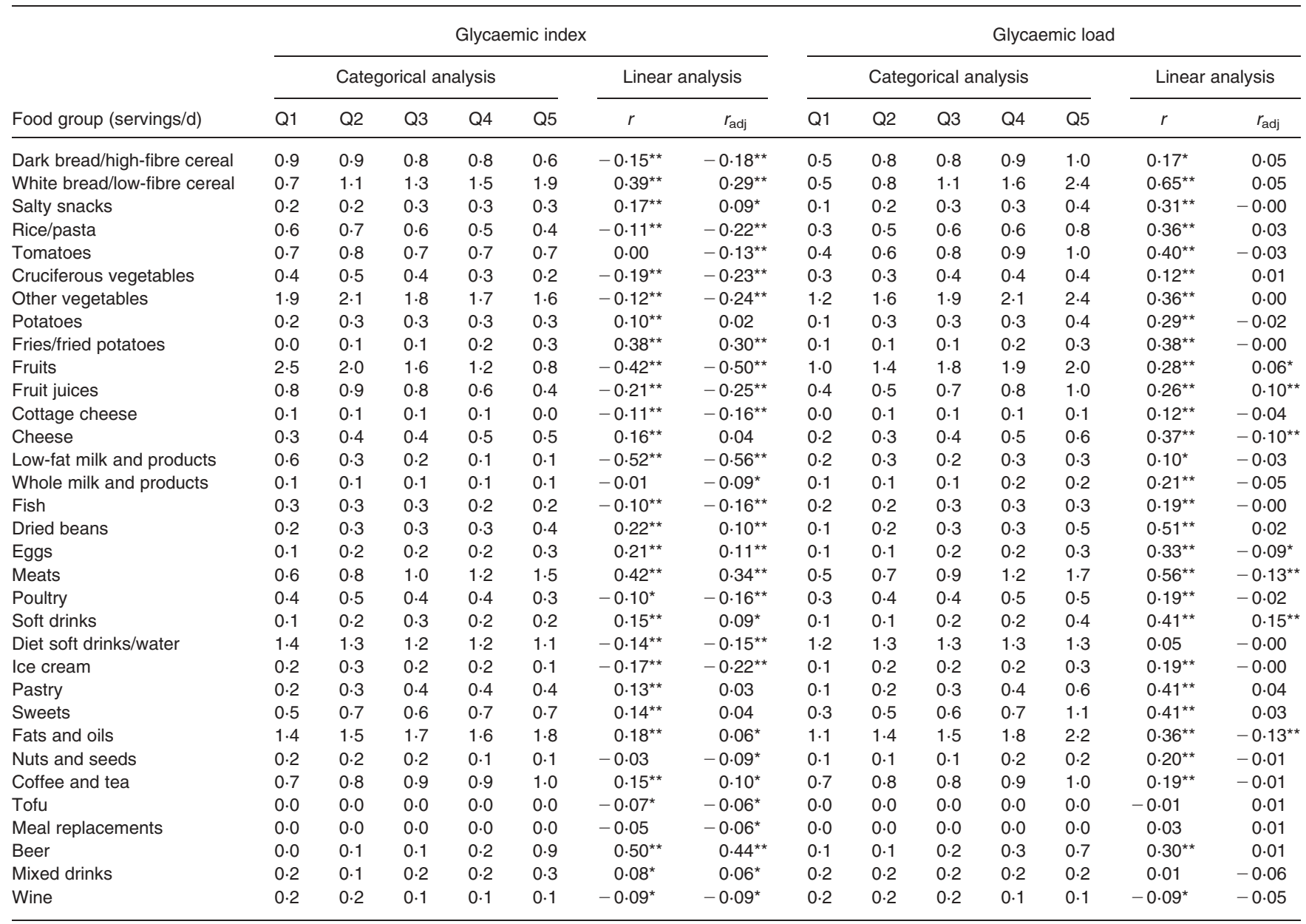

$r$, Pearson correlation coefficient; $r_{\text {adi }}$, correlation coefficient adjusted for total energy with residual method.

Correlation coefficients significant: ${ }^{\star} P<0.05,{ }^{\star \star} P<0.001$

†Based on glucose $=100$ scale.

‡ GI quintiles: Q1, <54.9; Q2, 54.9-57.0; Q3, 57.1-58.9; Q4, 60.0-61.1; Q5, >61.1. GL quintiles: Q1, <80.5; Q2, 80.5-105.8; Q3, 105.9-135.8; Q4, 135.9-167.3; Q5, >167.3. 
the correlation changed for carbohydrates $(-0 \cdot 27)$ and for protein $(-0.23)$, which means that under isoenergetic conditions, a high GI diet appeared higher in total fats but lower in digestible carbohydrates and protein compared with a low GI diet. Again, this effect may not be explained by collinearity between total energy intake and the nutrients (variance inflation factors $<10$ ). Correlation coefficients for fat and the fatty acids as well as for starch intake became weaker after adjusting for total energy, whereas the linear relationships between fibre $(-0.34)$ and sugars $(-0.30$ for sucrose to -0.52 for lactose) and dietary GI became stronger.

A slightly different picture was observed for the correlations between nutrients and dietary GL: while all the crude correlation coefficients were significantly positive, taking total energy into account revealed significantly negative correlations for total fat, fatty acids, lactose and protein. The remaining correlations were materially attenuated, except for alcohol. Correlation coefficients between nutrients and dietary GI/GL did not markedly vary by gender.

In order to allow direct comparison between observations made in the present study and current recommendations, we computed macronutrient intake data as a percentage of total energy. With regard to GI quintiles, mean values of percentage of energy were: $30 \cdot 6,32 \cdot 6,34 \cdot 7,36 \cdot 4$ and $36 \cdot 6$ for fat; $47 \cdot 9,46 \cdot 9,45 \cdot 5$, 43.5 and 41.2 for digestible carbohydrates; $17.7,16.6,16.0$, 16.2 and 15.2 for protein. In GL quintiles the mean values of percentage of energy were: $34.6,33.9,33.6,34.7$ and 34.4 for fat; $42.0,44.4,45.3,45.0$ and 45.5 for digestible carbohydrates; $17 \cdot 3,17 \cdot 0,16 \cdot 3,16 \cdot 1$ and $15 \cdot 6$ for protein.

\section{Prediction models}

We furthermore aimed to identify food groups that contributed to inter-individual variation in dietary GI and GL. The prediction models are shown by gender and were adjusted for age, clinic and ethnicity.

Among men, eight out of thirty-three food groups contributed significantly in explaining variation in GI (at least $1 \%$ ), accounting for $63 \%$ of GI variability after adjusting for demographic covariates (Table 4). The five food groups that contributed most to inter-individual variation in dietary GI were beer, low-fat milk and milk products, white bread/low-fibre cereal, fruits, and whole milk and milk products. While beer and white bread/lowfibre cereal were positively associated with dietary GI, the three other food groups were inversely associated. For instance, an increase of one serving of beer per day corresponded to an increase of 4.2 units in dietary GI, while an increase of one serving of low-fat milk per day corresponded to a decrease of $6 \cdot 0$ units in dietary GI. We detected some gender differences in the selection as well as in the ranking of the most predictive food groups. Among women, the prediction model selected eight food groups explaining $55 \%$ of variation in GI after adjusting for demographic covariates, with the five most predictive food groups being low-fat milk and milk products, fruits, white bread/low-fibre cereal, beer and potatoes. Compared with men, among women the predictive value of food groups was higher for low-fat milk and milk products, potatoes and for ice cream, for instance, and was lower for beer and fries/fried potatoes. Common to both genders was that low-fat milk and milk products as well as fruits were inversely associated, while beer and white bread/low-fibre cereal were positively associated with dietary GI.
The prediction models explaining variation in dietary GL showed marked differences in the selection and the importance of food groups compared with the GI prediction models. The model explaining variation in dietary GL (at least $1 \%$ ) among men selected eleven out of thirty-three food groups, which accounted for $70 \%$ of total GL variability after adjusting for demographic covariates (Table 5). The five food groups that contributed most to inter-individual variation in dietary GL were white bread/low-fibre cereal, dark bread/high-fibre cereal, regular soft drinks, beer and fruits. The highest increase in dietary GL was found for an increment of one serving of regular soft drinks and the lowest for a one-unit increment in consumption of fruit juices. The model among women selected nine of the thirty-three food groups, which explained $70 \%$ of total GL variation after adjusting for demographic covariates. The most predictive food groups were white bread/low-fibre cereal, rice/pasta, regular soft drinks, dark bread/high-fibre cereal and fruit juice. Compared with men, among women, an appreciably higher proportion of variation was explained by food groups such as rice/ pasta, fruit juice and pastry, and a lower proportion was explained, for instance, by the food groups dark bread/highfibre cereal and beer.

\section{Discussion}

The study of dietary GI in free-living populations requires consideration of the fact that foods are consumed in combination and consumption of foods is determined by many factors contributing to a very complex process. Therefore, in the present study, we related the wide variety of food groups and nutrients to dietary GI and GL, controlled for demographic factors. To date, no data on nutritional correlates have been reported from a multi-ethnic epidemiological study that has evaluated the broad range of dietary factors in relation to dietary GI and GL.

The most astounding finding was that the GI of a total diet, more so than the GL, was strongly associated with foods of low carbohydrate content and with non-carbohydrate nutrients. Dietary GL was, as expected, mostly associated with high-carbohydrate foods.

Foods with negligible amounts of carbohydrates being directly correlated with dietary GI were, for instance, meat, eggs, and fats and oils. Among these foods, meat exhibited the strongest positive correlation with dietary GI, which was entirely unexpected. A possible explanation for this finding could be that meat consumption is correlated with intake of other high GI foods such as white bread or potatoes. In previous work on dietary patterns in the same study population, we identified a specific dietary pattern, which was characterized by the high consumption of white bread, meat, tomatoes, cheese, beans, meat and beer (Liese et al. 2004b). To our knowledge there is no study that reported on the association between dietary GI and meat consumption.

A second interesting relation is the strong positive association between beer and dietary GI, which contrasts with the remarkably weak or null association of dietary GI with other alcoholic drinks. This finding may be driven by the GI value assigned to the alcoholic beverages. Beer has measurable amounts of starch, while the carbohydrate of wines and mixed drinks is limited to sucrose and the amount of carbohydrate is negligible as compared with beer. Because of its starch content, beer was assigned a value of 95 (glucose standard). Wine and mixed drinks were assigned a value of 61 , which corresponds to the GI of sucrose. Unlike the 


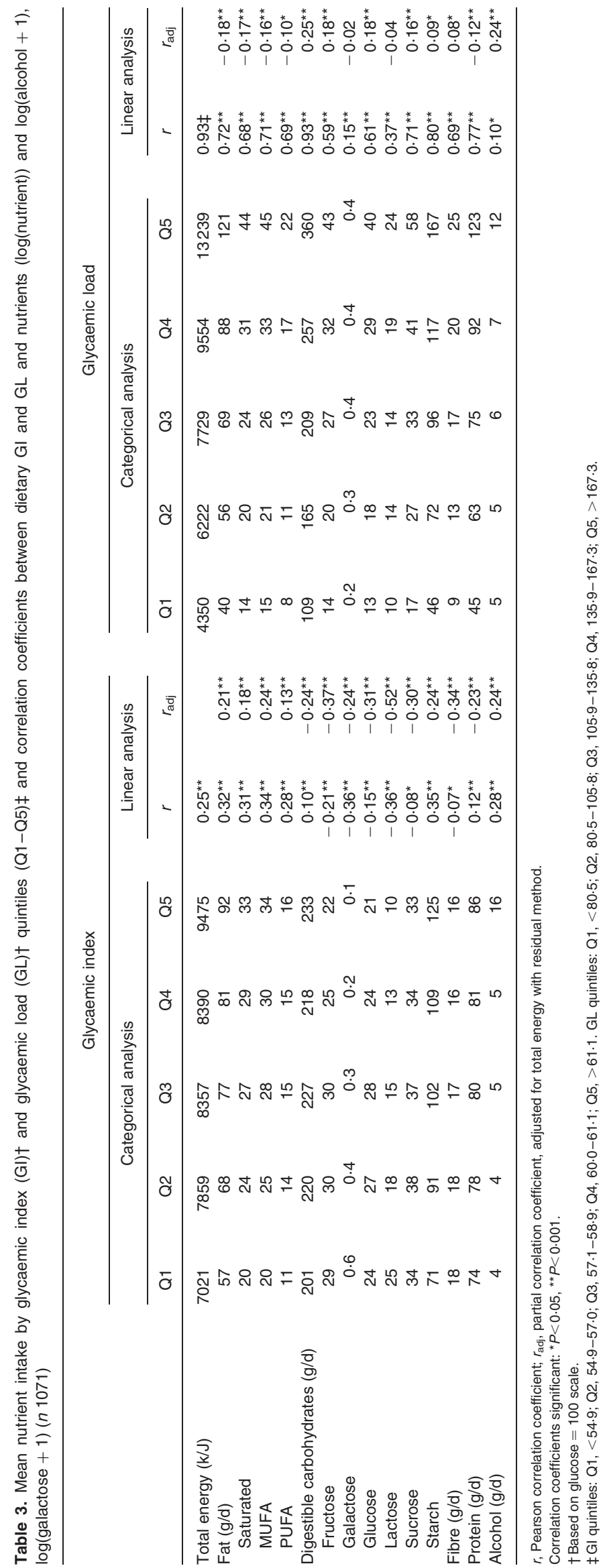


Table 4. Food groups $(\log ($ food group +1$))$ contributing* ${ }^{*}$ to inter-individua variation in average dietary glycaemic index† identified by stepwise linear regression, by gender $(n 1071)$

\begin{tabular}{lrcc}
\hline & $\beta$ & Partial $R^{2}$ & Model $R^{2}$ \\
\hline Men $(n$ 467) & & & $0.14 \S$ \\
Beer & 4.25 & 0.30 & 0.44 \\
Low-fat milk and products & -6.02 & 0.13 & 0.57 \\
White bread/low-fibre cereal & 3.14 & 0.08 & 0.64 \\
Fruits & -2.27 & 0.07 & 0.71 \\
Whole milk and products & -3.47 & 0.02 & 0.73 \\
Fries/fried potatoes & 2.20 & 0.02 & 0.75 \\
Dark bread/high-fibre cereal & 1.28 & 0.01 & 0.76 \\
Fruit juice & -1.12 & 0.01 & 0.77 \\
Women ( $n$ 604) & & & $0.18 \ddagger$ \\
Low-fat milk and products & -7.31 & 0.26 & 0.44 \\
Fruits & -2.65 & 0.10 & 0.54 \\
White bread/low-fibre cereal & 2.45 & 0.08 & 0.61 \\
Beer & 4.49 & 0.05 & 0.66 \\
Potatoes & 2.23 & 0.02 & 0.69 \\
Whole milk and products & -3.95 & 0.02 & 0.71 \\
Dark bread/high-fibre cereal & 1.27 & 0.01 & 0.72 \\
Ice cream & -2.13 & 0.01 & 0.73 \\
\hline
\end{tabular}

${ }^{*}$ Food groups explaining at least $1 \%$ of variation adjusted for demographics (age, clinic ethnicity).

$\dagger$ Based on glucose $=100$ scale

$\ddagger$ Explained variation by adjustment variables.

positive relationship between alcohol intake and dietary GI/GL observed in the present study, Michaud et al. (2002) and others (Salmerón et al. 1997a; Higginbotham et al. 2004; Schulze et al. 2004) found a decreasing trend of alcohol intake across dietary GI or GL quintiles. This inconsistency may have emerged from the fact that we assigned GI values to alcoholic beverages while

Table 5. Food groups $(\log ($ food group +1$))$ contributing $^{*}$ to inter-individual variation in average dietary glycaemic load $\dagger$ identified by stepwise linear regression, by gender $(n 1071)$

\begin{tabular}{lccc}
\hline & $\beta$ & Partial $R^{2}$ & Model $R^{2}$ \\
\hline Men ( $n$ 467) & & & $0.18 \S$ \\
White bread/low-fibre cereal & 57.22 & 0.32 & 0.50 \\
Dark bread/high-fibre cereal & 23.79 & 0.09 & 0.59 \\
Regular soft drinks & 87.16 & 0.08 & 0.66 \\
Beer & 33.30 & 0.05 & 0.71 \\
Fruits & 23.66 & 0.04 & 0.76 \\
Sweets/sugar & 22.79 & 0.04 & 0.80 \\
Rice/pasta & $33 \cdot 20$ & 0.03 & 0.83 \\
Pastry & 26.05 & 0.02 & 0.85 \\
Salty snacks & 27.67 & 0.01 & 0.86 \\
Fruit juice & 22.29 & 0.01 & 0.87 \\
Fries/fried potatoes & 35.93 & 0.01 & 0.88 \\
Women ( $n$ 604) & & & $0.17 \ddagger$ \\
White bread/low-fibre cereal & 38.04 & 0.27 & 0.44 \\
Rice/pasta & 28.16 & 0.12 & 0.56 \\
Regular soft drinks & 69.24 & 0.09 & 0.65 \\
Dark bread/high-fibre cereal & 23.93 & 0.07 & 0.73 \\
Fruit juice & 19.29 & 0.04 & 0.77 \\
Pastry & 27.86 & 0.04 & 0.81 \\
Fruits & 10.37 & 0.03 & 0.84 \\
Dried beans & 27.86 & 0.02 & 0.86 \\
Fries/fried potatoes & 44.73 & 0.01 & 0.87 \\
\hline Fil & & & \\
\hline
\end{tabular}

*Food groups explaining at least $1 \%$ of variation adjusted for demographics (age, clinic, ethnicity).

† Based on glucose $=100$ scale

$\ddagger$ Explained variation by adjustment variables. other investigators considered alcoholic beverages as low-carbohydrate foods and thus did not include these items in the estimation of dietary GI. However, we can only speculate on this, since previous studies have provided very limited detail of assigning GI values to food items.

With regard to macronutrients, we observed that a high-GI diet was characterized by high fat, low carbohydrate and low protein intake, compared with a low-GI diet. This is in agreement with previous studies that reported an inverse association of dietary GI with total carbohydrates (Jonas et al. 2003; Scholl et al. 2004; Schulze et al. 2004). In previous studies, results regarding fat intake and dietary GI were equivocal: while one study (Buyken et al. 2001) observed a decreasing trend of animal fat intake across GI quintiles, two other studies (Jonas et al. 2003; Scholl et al. 2004) reported a positive trend of fat intake.

Focusing on the carbohydrate fractions, it became apparent that a high-GI diet was positively correlated with starch (carbohydrates, for example, in grains and potatoes), whereas the mono- and disaccharides (carbohydrates in fruits and milk/milk products) as well as fibre intake showed inverse associations with dietary GI. Consequently, in our study population, a highGI diet was high in starch intake but low in sugar intakes compared with a low-GI diet, which resulted in an overall decrease in the amount of total carbohydrates. This is in perfect agreement with the high intake of white bread (a food group that was found to be positively associated with dietary GI) and the low intake of fruits and milk products (food groups that were found to be negatively associated with dietary GI) in the high-GI diet. Our findings are in agreement with previous studies that reported an inverse association of dietary GI with simple sugars (Wolever et al. 1994) and fibre (Jonas et al. 2003; Scholl et al. 2004; Schulze et al. 2004) and direct associations for starchy foods (Salmerón et al. 1997a; Buyken et al. 2001).

We could confirm a positive association of potatoes with dietary GI observed in previous studies (Salmerón et al. 1997a; Buyken et al. 2001). However, in the present study consumption of potatoes was less important for dietary GI and GL than consumption of many other food groups, especially among men. Differences in eating patterns between study populations may explain the inconsistency in this association: while in many European regions cooked potatoes are more commonly consumed than fried potatoes, the consumption of French fries and fried potatoes may be favoured over cooked potatoes in our US study population. A positive correlation between French fries and dietary GI has also been shown in a US population of male health professionals (Salmerón et al. 1997).

Dietary GI was associated with a number of demographic and socio-economic characteristics. In the multi-ethnic IRAS population, a significantly larger proportion of Hispanics consumed a diet high in GI or GL compared with the non-Hispanic white or African American population. Similarly to the ARIC study (Stevens et al. 2002), we also found that women tended to eat diets lower in GI than men. Additionally, consumption of diets higher in GI or GL was associated with indicators of lower socio-economic status, such as lower levels of education and larger prevalence of smoking. Similar trends with regard to smoking were observed in the African American group of the population-based ARIC study, and with regard to education in both Whites and African Americans in ARIC (Stevens et al. 2002), but opposing trends were observed in two populations of highly educated health professionals (Salmerón et al. 1997a,b). 
The consideration of demographic characteristics in GI research in free-living individuals merits special attention. As indicators of diet and lifestyle, on the one hand, and of some genetic background, on the other hand, they may confound the association between dietary GI and an outcome.

The application of GI to mixed meals and total diet deserves closer attention. Although much controversy exists about the accuracy of GI for mixed meals, and actual metabolic responses in free-living individuals consuming food in combination may not confirm the GI values of foods obtained from tests (Laine et al. 1987; Schrezenmeir et al. 1989; Hollenbeck \& Coulston, 1991; Flint et al. 2004), it has been demonstrated that the glycaemic response to mixed meals can reasonably accurately be predicted from the GI of the constituent foods (Wolever \& Jenkins, 1986; Chew et al. 1988; Wolever \& Bolognesi, 1996). Evidence is limited as to the applicability of GI to overall diet. Even though in a short-term intervention trial the effect of a low-GI diet was demonstrated on concentrations of C-peptide (Jenkins et al. 1987), it has to be acknowledged that differences may exist in total diets administered under experimental conditions and in free-living individuals. In an intervention setting, low- and high-GI diets differ only in the quality of carbohydrate foods consumed (e.g. whole-grain kernels $v$. refined grains, or pasta $v$. mashed potatoes; Heilbronn et al. 2002; Frost et al. 2004; Sloth et al. 2004). They usually do not differ in other foods and in energy and macronutrient composition. In the present study, we found all these differences, apart from the selection of carbohydrate, in a diverse, free-living population. Obviously, this would have implications for the interpretation of dietary GI in epidemiological studies, since any effect seen for dietary GI on an outcome may be difficult to interpret without understanding nutritional correlates of dietary GI.

Along these lines, the question arises as to the applicability of the concept of GI to characterize a long-term dietary exposure. The GI of a food describes an acute physiological event $(2 \mathrm{~h}$ postprandial glucose response) provoked under controlled experimental conditions. The chronic consumption of high-GI foods is thought to challenge glucose homeostasis mechanisms and, consequently, may lead to insulin resistance and other related conditions (Ludwig, 2002). Effects on glucose and insulin regulatory mechanisms have been demonstrated in some experimental studies (Jenkins et al. 1987; Wolever et al. 1992; Schafer et al. 2003; Rizkalla et al. 2004), but not in others (Kiens \& Richter, 1996; Sloth et al. 2004). Long-term evidence is only provided by observational studies relating dietary GI to incidence of type 2 diabetes mellitus. Also here, results are equivocal: while some studies detected an association (Salmerón et al. 1997a,b; Schulze et al. 2004), others did not (Meyer et al. 2000; Stevens et al. 2002). Thus, the impact of dietary GI as a long-term dietary exposure remains questionable and warrants future research. Certainly, chronic exposure to high glucose responses may have implications for other metabolic processes, but it needs to be recognized that GI is only one characteristic of diet (and lifestyle) and effects may be both reduced or magnified due to other conditions such as adiposity and physical activity (Willett et al. 2002).

The conceptual framework of our present study merits special attention. We paid significant attention to the assignment of GI values to foods assessed in IRAS. We considered nutritional correlates of GI that have previously not been described or taken into account (either due to negligible amounts of carbohydrates, such as meat, milk and cheese, or due to omitting specific items, such as beer). It needs to be recognized that this leads to an important distinction in our study findings: while some associations observed between nutritional factors and dietary GI/GL are driven by the fact that the food contributes significantly to total energy intake and also has a particularly high or low GI (such as bread or milk), other associations are purely coincidental (such as meat or fat). These correlations may have emerged due to the fact that people who consume large amounts of high-GI foods also tend to eat a lot of fat. A strength of our study is that we used diet data from a validated instrument, which has been shown to detect diet-disease relationships (Mayer-Davis et al. 1997a; Bell et al. 2000; Liese et al. 2003).

A limitation of the present study is that our FFQ, similar to most epidemiological studies to date, has not been designed specifically to measure dietary GI or GL. Consequently, our assignment of GI values was conducted within the constraints of the FFQ, which included that GI values for those food lines containing multiple foods were computed by an averaging or weighting procedure. The satisfactory validity of carbohydrate intake in our study population (Mayer-Davis et al. 1999) that is comparable to other work in multi-ethnic populations provides some reassurance. In future studies, however, the focus should be directed toward the validity of dietary GI in epidemiological studies. A second limitation refers to the fact that our observations might be of limited value regarding inferences to other study populations in that the findings of the present study may not be reproducible in different study populations, e.g. children or elderly people. However, they may translate well to middleaged US-American populations considering the multi-centre design of the IRAS.

In conclusion, we identified carbohydrate as well as non-carbohydrate foods to be significantly related to dietary GI. Although the GI value of a food is an indicator of the ability of carbohydrates to raise blood glucose, average GI of diet appears to reflect more dimensions of diet than just quality of carbohydrates, such as the combination of foods consumed. Given the diverse associations between food group consumption and dietary GI that we found in the present study, the currently widely used definition of dietary GI in terms of overall quality of carbohydrates of diet may be reconsidered and raised to a broader understanding, including other dietary factors such as (animal) fat intake and alcohol consumption. This may have implications for the interpretation of dietary GI in epidemiological studies.

\section{Acknowledgements}

This study was supported by NIH/NHLBI grants UO1 HL/17887, UO1 HL/17889, UO1 HL/17890, UO1 HL/17892, UO1 HL/ 17902 and DK29867 and by an American Diabetes Association Clinical Research Award to Dr Angela Liese.

\section{References}

Augustin LS, Franceschi S, Jenkins DJ, Kendall CW \& La Vecchia C (2002) Glycemic index in chronic disease: a review. Eur J Clin Nutr 56, 1049-1071.

Bell RA, Mayer-Davis EJ, Martin MA, D'Agostino RB \& Haffner SM (2000) Associations between alcohol consumption and insulin sensitivity and cardiovascular disease risk factors: the Insulin Resistance and Atherosclerosis Study. Diabetes Care 23, 1630-1636. 
Block G, Hartman A, Dresser C, Carroll MD, Gannon J \& Gardner L (1986) A data-based approach to diet questionnaire design and testing. Am J Epidemiol 124, 453-469.

Buyken AE, Toeller M, Heitkamp G, Karamonos B, Rottiers R, Muggeo M \& Fuller JH (2001) Glycemic index in the diet of European outpatients with type 1 diabetes: relations to glycated hemoglobin and serum lipids. Am J Clin Nutr 73, 574-581.

Chew I, Brand JC, Thorburn AW \& Truswell AS (1988) Application of glycemic index to mixed meals. Am J Clin Nutr 47, 53-56.

Crapo PA, Insel J, Sperling M \& Kollerman DG (1981) Comparison of serum glucose, insulin, and glucagon responses to different types of complex carbohydrate in noninsulin-dependent diabetic patients. Am J Clin Nutr 34, 184-190.

Englyst HN \& Cummings JH (1986) Digestion of the carbohydrates of banana (Musa paradisiaca sapientum) in the human small intestine. Am J Clin Nutr 44, 42-50.

Ercan N, Gannon MC \& Nuttall FQ (1994) Effect of added fat on the plasma glucose and insulin response to ingested potato given in various combinations as two meals in normal individuals. Diabetes Care 17, $1453-1459$.

Flint A, Moller BK, Raben A, Pedersen D, Tetens I, Holst JJ \& Astrup A (2004) The use of glycaemic index tables to predict glycaemic index of composite breakfast meals. Br J Nutr 91, 979-989.

Foster-Powell K, Holt SH \& Brand-Miller JC (2002) International table of glycemic index and glycemic load values: 2002. Am J Clin Nutr 76, 5-56.

Frost GS, Brynes AE, Bovill-Taylor C \& Dornhorst A (2004) A prospective randomised trial to determine the efficacy of a low glycaemic index diet given in addition to healthy eating and weight loss advice in patients with coronary heart disease. Eur J Clin Nutr 58, 121-127.

Heilbronn LK, Noakes M \& Clifton PM (2002) The effect of high- and low-glycemic index energy restricted diets on plasma lipid and glucose profiles in type 2 diabetic subjects with varying glycemic control. J Am Coll Nutr 21, 120-127.

Higginbotham S, Zhang ZF, Lee IM, Cook NR, Buring JE \& Liu S (2004) Dietary glycemic load and breast cancer risk in the Women's Health Study. Cancer Epidemiol Biomarkers Prev 13, 65-70.

Hollenbeck CB \& Coulston AM (1991) The clinical utility of the glycemic index and its application to mixed meals. Can J Physiol Pharmacol 69, 100-107.

Jenkins DJ, Wolever TM, Collier GR, Ocana A, Rao AV, Buckley G, Lam Y, Mayer A \& Thompson LU (1987) Metabolic effects of a low-glycemic-index diet. Am J Clin Nutr 46, 968-975.

Jenkins DJ, Wolever TM, Jenkins AL, Thorne MS, Lee R, Kalmusky J, Reicherst R \& Wong GS (1983) The glycaemic index of foods tested in diabetic patients: a new basis for carbohydrate exchange favouring the use of legumes. Diabetologia 24, 257-264.

Jenkins DJ, Wolever TM, Taylor RH, Barker H, Fielden H, Baldwin JM, Bowling AC, Newman HC, Jenkins AL \& Goff DV (1981) Glycemic index of foods: a physiological basis for carbohydrate exchange. Am J Clin Nutr 34, 362-366.

Jonas CR, McCullough ML, Teras LR, Nalker-Thurmond A, Thun MJ \& Calle EE (2003) Dietary glycemic index, glycemic load, and risk of incident breast cancer in postmenopausal women. Cancer Epidemiol Biomarkers Prev 12, 573-577.

Kiens B \& Richter EA (1996) Types of carbohydrate in an ordinary diet affect insulin action and muscle substrates in humans. Am J Clin Nutr 63, 47-53.

Laine DC, Thomas W, Levitt MD \& Bantle JP (1987) Comparison of predictive capabilities of diabetic exchange lists and glycemic index of foods. Diabetes Care 10, 387-394.

Liese AD, Fang F, Schulz M, et al. (2004a) No association of glycemic index with insulin sensitivity and adiposity in IRAS. Diabetes 53, Suppl. 2, A589.

Liese AD, Roach AK, Sparks KC, Marquart L, D’Agostino RB \& MayerDavis EJ (2003) Whole-grain intake and insulin sensitivity: the Insulin Resistance Atherosclerosis Study. Am J Clin Nutr 78, 965-971.
Liese AD, Schulz M, Moore CG \& Mayer-Davis EJ (2004b) Dietary patterns, insulin sensitivity, and adiposity in the multi-ethnic Insulin Resistance Atherosclerosis Study population. Br J Nutr 92, 973-984.

Ludwig DS (2002) The glycemic index: physiological mechanisms relating to obesity, diabetes, and cardiovascular disease. JAMA 287, 2414-2423.

Mayer-Davis EJ, Costacou T, King I, Zaccaro DJ \& Bell RA (2002) Plasma and dietary vitamin $\mathrm{E}$ in relation to incidence of type 2 diabetes: the Insulin Resistance and Atherosclerosis Study (IRAS). Diabetes Care 25, 2172-2177.

Mayer-Davis EJ, Monaco JH, Hoen HM, Carmichael S, Virolins MZ, Rewers MJ, Haffner SM, Ayed MF, Bergman GN \& Karter AJ (1997a) Dietary fat and insulin sensitivity in a triethnic population: the role of obesity. The Insulin Resistance Atherosclerosis Study (IRAS). Am J Clin Nutr 65, 79-87.

Mayer-Davis EJ, Monaco JH, Marshall JA, Rushing J \& Juhaeri A (1997b) Vitamin C intake and cardiovascular disease risk factors in persons with non-insulin-dependent diabetes mellitus. From the Insulin Resistance Atherosclerosis Study and the San Luis Valley Diabetes Study. Prev Med 26, 277-283.

Mayer-Davis EJ, Vitolins MZ, Carmichael SL, Hemphill S, Tsaroucha E, Rushing J \& Levin S (1999) Validity and reproducibility of a food frequency interview in a Multi-Cultural Epidemiology Study. Ann Epidemiol 9, 314-324.

Meyer KA, Kushi LH, Jacobs DR, Slavin J, Sellers TA, Folsom AR \& Jacobs DRJ (2000) Carbohydrates, dietary fiber, and incident type 2 diabetes in older women. Am J Clin Nutr 71, 921-930.

Michaud DS, Liu S, Giovannucci E, Willelt WC, Coldite GA \& Fuchs CS (2002) Dietary sugar, glycemic load, and pancreatic cancer risk in a prospective study. J Natl Cancer Inst 94, 1293-1300.

O’Dea K, Nestel PJ \& Antonoff L (1980) Physical factors influencing postprandial glucose and insulin responses to starch. Am J Clin Nutr 33, 760-765.

Rizkalla SW, Taghrid L, Laromiguiere M, Huet D, Boillot J, Rigoir A, Elgrably F \& Slama G (2004) Improved plasama glucose control, whole-body glucose utilization, and lipid profile on a low-glycemic index diet in type 2 diabetic men. Diabetes Care 27, 1866-1872.

Salmerón J, Ascherio A, Rimm EB, Colditz GA, Spiegelman D, Jenkins DJ, Stampfer MJ, Wing AL \& Willett WC (1997a) Dietary fiber, glycemic load, and risk of NIDDM in men. Diabetes Care 20, 545-550.

Salmerón J, Manson JE, Stampfer MJ, Colditz GA, Wing AL \& Willett WC (1997b) Dietary fiber, glycemic load, and risk of non-insulindependent diabetes mellitus in women. JAMA 277, 472-477.

Sanchez-Lugo L, Mayer-Davis EJ, Howard G, Selby JV, Ayad MF, Rewers M \& Haffner S (1997) Insulin sensitivity and intake of vitamins $\mathrm{E}$ and $\mathrm{C}$ in African American, Hispanic, and non-Hispanic white men and women: the Insulin Resistance and Atherosclerosis Study (IRAS). Am J Clin Nutr 66, 1224-1231.

Schafer G, Schenk U, Ritzel U, Ramadari G \& Leonhardt I (2003) Comparison of the effects of dried peas with those of potatoes in mixed meals on postprandial glucose and insulin concentrations in patients with type 2 diabetes. Am J Clin Nutr 78, 99-103.

Scholl TO, Chen X, Khoo CS \& Lenders C (2004) The dietary glycemic index during pregnancy: influence on infant birth weight, fetal growth, and biomarkers of carbohydrate metabolism. Am J Epidemiol 159, 467-474.

Schrezenmeir J, Tato F, Tato S, Kustner E, Krause U, Hommel G, Asp NG, Kasper H \& Beyer J (1989) Comparison of glycemic response and insulin requirements after mixed meals of equal carbohydrate content in healthy, type-1, and type-2 diabetic man. Klin Wochenschr 67, 985-994.

Schulze MB, Liu S, Rimm EB, Manson JE, Willett WC \& Hu FB (2004) Glycemic index, glycemic load, and dietary fiber intake and incidence of type 2 diabetes in younger and middle-aged women. Am J Clin Nutr 80, 348-356

Sloth B, Krog-Mikkelsem I, Flint A, Tetens I, Bjorck I, Vinoy S, Elarstahl H, Astrup A, Lang V, \& Raben A (2004) No difference in body weight decrease between a low-glycemic-index 
and a high-glycemic-index diet but reduced LDL cholesterol after 10wk ad libitum intake of the low-glycemic index diet. Am J Clin Nutr 80, 337-347.

Stevens J, Ahn K, Juhaeri, Houston D, Steffan L \& Couper D (2002) Dietary fiber intake and glycemic index and incidence of diabetes in African-American and white adults: the ARIC study. Diabetes Care 25, $1715-1721$.

Vaaler S, Hanssen KF \& Aagenaes O (1984) The effect of cooking upon the blood glucose response to ingested carrots and potatoes. Diabetes Care 7, 221-223.

Wagenknecht LE, Mayer EJ, Rewers M, et al. (1995) The Insulin Resistance Atherosclerosis Study (IRAS) objectives, design, and recruitment results. Ann Epidemiol 5, 464-472.

Willett W, Manson J \& Liu S (2002) Glycemic index, glycemic load, and risk of type 2 diabetes. Am J Clin Nutr 76, 274S-280S.

Willett W \& Stampfer MJ (1986) Total energy intake: implications for epidemiologic analyses. Am J Epidemiol 124, 17-27.

Wolever TM \& Bolognesi C (1996) Prediction of glucose and insulin responses of normal subjects after consuming mixed meals varying in energy, protein, fat, carbohydrate and glycemic index. J Nutr 126, 2807-2812.

Wolever TM \& Jenkins DJ (1986) The use of the glycemic index in predicting the blood glucose response to mixed meals. Am J Clin Nutr 43, 167-172. Wolever TM, Jenkins DJ, Vuksan V, Josse RG, Wong GS \& Jenkins AL (1990) Glycemic index of foods in individual subjects. Diabetes Care 13, 126-132.

Wolever TM, Jenkins DJ, Vuksan V, Josse RG, Wong GS \& Jenkins AL (1992) Beneficial effect of low-glycemic index diet in overweight NIDDM subjects. Diabetes Care 15, 562-564.

Wolever TMS, Nuttall FQ, Lee R, Wong GS, Josse RG, Csima A \& Jenkins DJ (1985) Prediction of the relative blood glucose response of mixed meals using the white bread glycaemic index. Diabetes Care 8, 418-428.

Wolever TM, Nguyen PM, Chiasson JL, Hunt JA, Josse RG, Palmason C, Rodger NW, Ross SA, Ryan EA \& Tan MH (1994) Determinants of diet glycemic index calculated retrospectively from diet records of 342 individuals with non-insulin-dependent diabetes mellitus. Am J Clin Nutr 59, 1265-1269. 